\title{
Modelación empírica de flotación en columna a escala piloto ${ }^{(\bullet)}$
}

\author{
Resumen

\begin{abstract}
El área Procemin I, del Centro Minero Metalúrgico Tecnología y Servicio (CIMM T\&S), ubicado en Santiago de Chile, cuenta con una completa planta piloto de molienda y flotación en la cual se desarrollan experiencias de optimización de circuitos, diseño de plantas, obtención de parámetros de operación, entre otras cosas. Uno de los equipos que posee la planta piloto es una columna de flotación piloto, con un alto nivel de automatización. Una de las problemáticas presentadas para la operación de la columna de flotación es no lograr tomar decisiones de operación sobre la base de los resultados metalúrgicos que se puedan obtener. El desarrollo de modelos matemáticos que permitan estimar los resultados metalúrgicos (ley de concentrado, ley de relaves, razón de enriquecimiento y recuperación) en función de las variables operacionales que sean manipulables busca entregarle mayor libertad al operador y al ingeniero de procesos para lograr la sintonización operacional de la columna. El método empleado para el desarrollo de los modelos fue Stepwise Regression (Regresión por Pasos) considerando relaciones empíricas entre variables operacionales y los resultados experimentales. Todos los modelos presentaron sobre un $90 \%$ de exactitud, salvo uno de ellos que fue sobre $70 \%$.
\end{abstract}

J. Simpson*, D. Jordán*, G. Cifuentes*, A. Morales** y L. Briones***

\section{Mathematical simulation of column flotation in pilot scale}

\begin{abstract}
The Procemin-I area of the Centro Minero Metalúrgico Tecnología y Servicio (CIMM T \& S), has a full milling and flotation pilot plant in which several experiences are developed as: optimization of circuits, plant design, procurement of operating parameters, etc. Ones of the equipment in operation is the column flotation to pilot scale, witch have a medium level of automation. The problem presented in the operation of the column flotation is the low relationship during the operation between the operating basis parameters and the metallurgical results. The mathematical models used today to estimate the metallurgical results (i.e.: concentrate, tailing, enrichment and recovery) depending on variables that are manipulated by hand according the operator experience. But the process engineer needs tools without subjective vision to obtain the best performance of the column. The method used to help the column operation was a mathematical model based on the Stepwise Regression then considering empirical relationships between operational variables and experimental results. All the mathematicals relationship developed in this study have a good correlation (up $90 \%$ of precision), except one (up $70 \%$ ) due by non regular mineralogical feed.
\end{abstract}

Keyword

Float in column; Stepwise regression; Mathematical modelling.

\section{ANTECEDENTES}

Los orígenes de la columna de flotación se remontan a principios del siglo XX, cuando se presentaba la máquina de flotación de Callow ${ }^{[1]}$, una máquina simple que consistía en una celda en la cual se reemplazaba la agitación mecánica por una columna de burbujas producidas por inyección de aire a presión, el cual pasaba por una tela porosa produciendo burbujas de aire que colectaban las partículas mineralizadas e hidrofobizadas, las cuales ascendían hasta la parte superior de la celda para ser retiradas como concentrado.

El diseño anterior inspiró a D. Wheeler, P. Bouitn y R. Tremblay ${ }^{[2]}$ quienes, en 1960, diseñaron una celda de flotación basándose en el principio de Callow, generar la fase gaseosa mediante inyección de aire en la base de la celda. Esta nueva celda poseía como características principales una gran altura, se alimentaba desde la parte superior (a $2 / 3$ de la altura total)

(•) Trabajo recibido el día 13 de agosto de 2008 y aceptado en su forma final el día 2 de diciembre de 2009.

* Dpto. de Ingeniería Metalúrgica, Universidad de Santiago de Chile, Santiago, Chile.

** Dpto. de Ingeniería Metalúrgica, Universidad Católica del Norte, Antofagasta, Chile.

*** Dpto. de Ingeniería Mecánica, Universidad de Santiago de Chile, Santiago, Chile.gerardo.cifuentes@usach.cl. 
generando un flujo en contra-corriente con un flujo descendente de pulpa mineral y un flujo ascendente de burbujas de aire, y un pequeño flujo de agua sobre el lecho de espuma a fin de limpiar el concentrado. Alrededor de $1970^{[2]}$, en la Canada Iron Company realizaron las primeras pruebas en estos equipos, obteniendo resultados metalúrgicos satisfactorios. Aún cuando el equipo contenía un sinnúmero de aspectos ventajosos: menor espacio ocupado, menores costos de operación por eliminación de la agitación mecánica, mejores resultados metalúrgicos; el proyecto se enfrentó al conservadurismo de las empresas metalúrgicas proveedoras de celdas de flotación. En la actualidad la utilización de columnas de flotación en los procesos metalúrgicos en la industria del cobre es una realidad, con una utilidad más que demostrada y avalada económicamente.

Un aspecto importante dentro de todo proceso es el control de éste y la operación de las columnas no está ajena a esta realidad; en este trabajo se desarrolla un modelo matemático basado en el método de regresión por pasos (Stepwise Regression). Este método trabaja bajo la hipótesis de que la relación entre las variables predictoras y la variable respuesta es lineal; de esta forma, se elige un subconjunto de variables que deben estar incluidas en el modelo. En la regresión por pasos, al incluir una nueva variable al modelo, reconsidera el mantener las que ya se habían añadido previamente, es decir, no sólo puede entrar una nueva variable en cada paso sino que puede salir alguna de las que ya estaban en la ecuación. El proceso finaliza cuando ninguna variable de las que no están en la ecuación cumple la condición para entrar y, de las incorporadas a la ecuación, ninguna cumple la condición de salir. El criterio que se emplea para decidir si una variable se queda o no corresponde, estadísticamente, al coeficiente de regresión lineal ( $\mathrm{R}$ o $\mathrm{R}^{2}$ ) calculado cada vez que una variable se incorpora o se quita del modelo. El valor, por ejemplo, de $\mathrm{R}^{2}$ mide la proporción de la variabilidad de la variable dependiente en la ecuación del modelo planteado.

Las variables que pueden ser incorporadas en el método de regresión por pasos pueden tener un origen muy variado, pasando desde variables empíricas hasta del tipo fenomenológico. Cuando la mayoría de las variables son del tipo empíricas se tendrá como resultado un modelo empírico. En cambio, cuando las variables incorporadas en el modelo poseen componentes empíricas y fenomenológicas estamos en presencia de un modelo gris. En este trabajo se pretende desarrollar un modelo matemático utilizando las herramientas estadísticas que se desprenden del método de regresión por pasos.

\section{OBJETIVOS}

Se pretende obtener un modelo matemático por el método de regresión por pasos representativo, en estado estacionario, que determine los resultados metalúrgicos de la columna de flotación piloto en función de los parámetros de operación.

\section{METODOLOGÍA}

Se realizó una caracterización preliminar del mineral empleado en este trabajo, utilizando nueve muestras. En primer lugar, se caracterizó químicamente, mostrándose los resultados obtenidos en la tabla I para, después, proceder a realizar una flotación Rougher estándar en celda convencional con el fin de obtener la constante cinética de flotación, $\mathrm{K}_{\text {Rougher, }}$ y la recuperación infinita, $R_{\infty}$, según el método de Klimpell[ ${ }^{[3]}$ para proceder al escalamiento a flotación columnar. En la tabla II, se presentan los valores cinéticos correspondientes.

Tabla I. Análisis químicos a muestras representativas del mineral ensayado

Table I. Chemical analysis of representative samples ore tested

\begin{tabular}{lrrrrrrrrr}
\hline & \multicolumn{8}{c}{ Muestras } \\
\cline { 2 - 10 } \multicolumn{1}{c}{ Elemento } & \multicolumn{1}{c}{ M1 } & M2 & M3 & M4 & M5 & M6 & M7 & M8 & M9 \\
\hline Cu, \% & 1,85 & 1,79 & 1,79 & 1,01 & 1,40 & 1,35 & 1,31 & 0,97 & 1,47 \\
Insolubles, \% & 82,30 & 80,80 & 80,80 & 83,90 & 84,73 & 81,95 & 82,27 & 83,33 & 85,12 \\
S, \% & 4,80 & 4,47 & 4,47 & 3,72 & 3,87 & 3,88 & 3,86 & 3,33 & 2,47 \\
Fe, \% & 3,37 & 3,45 & 3,45 & 2,92 & 3,05 & 3,65 & 3,84 & 3,20 & 2,88 \\
As, \% & 0,13 & 0,16 & 0,16 & 0,07 & 0,12 & 0,08 & 0,08 & 0,05 & 0,12 \\
\hline
\end{tabular}


Tabla II. Parámetros cinéticos obtenidos en flotación Rougher estándar en celda convencional

Table II. Kinetic parameters obtained in Rougher flotation conventional standard cell

\begin{tabular}{ccc}
\hline \multirow{2}{*}{ Muestras } & \multicolumn{2}{c}{ Parámetros cinéticos } \\
\cline { 2 - 3 } & $\mathbf{K}_{\text {Rougher }}$ & $\mathbf{R}_{\infty}$ \\
\hline M1 & 1,10 & 99,8 \\
M2 & 0,80 & 98,5 \\
M3 & 0,80 & 98,5 \\
M4 & 0,75 & 98,1 \\
M5 & 0,30 & 86,1 \\
M6 & 0,30 & 100 \\
M7 & 0,40 & 100 \\
M8 & 0,30 & 100 \\
M9 & 0,20 & 87,4 \\
\hline
\end{tabular}

Lo más notorio, en la tabla II, es la dispersión de valores obtenidos en los parámetros cinéticos para las muestras representativas, aspecto que tiene que ver más bien con la dispersión del tipo mineralógica presente en el mineral, que con el contenido químico del elemento presente en este que, como lo presenta la tabla I, no están tan dispersos respecto del cobre principalmente, no así del contenido de azufre que podría indicar diferencias significativas en cuanto a las variables cinéticas, al estar asociado a diferentes especies mineralógicas con el cobre.

La metodología de trabajo consistió en recopilar la información operacional de nueve ensayos de la planta de procesamiento de minerales. La información fue recopilada empleando un software de adquisición de datos desde la instrumentación asociada a la columna de flotación piloto (Fig. 1). El lazo de control de estabilización de la columna de la figura 1 emplea sólo el control de nivel, siendo este tipo de lazo una de las alternativas de más bajo costo de implementación.

Los sensores empleados en este lazo fueron: sensores de presión (LT) dPcell instalados en la pared de la columna y columna de burbujeo o presión estática; sensores de temperatura (FT) para determinar la posición de la interfaz pulpa/espuma por diferencia entre la temperatura del agua de lavado y la pulpa; sensores ultrasónicos y de flotación para determinar la profundidad de la espuma; y censor radiactivo (C) para determinar la densidad de pulpa de alimentación.

En la tabla III se presenta la simbología empleada para las diferentes variables asociadas a la operación

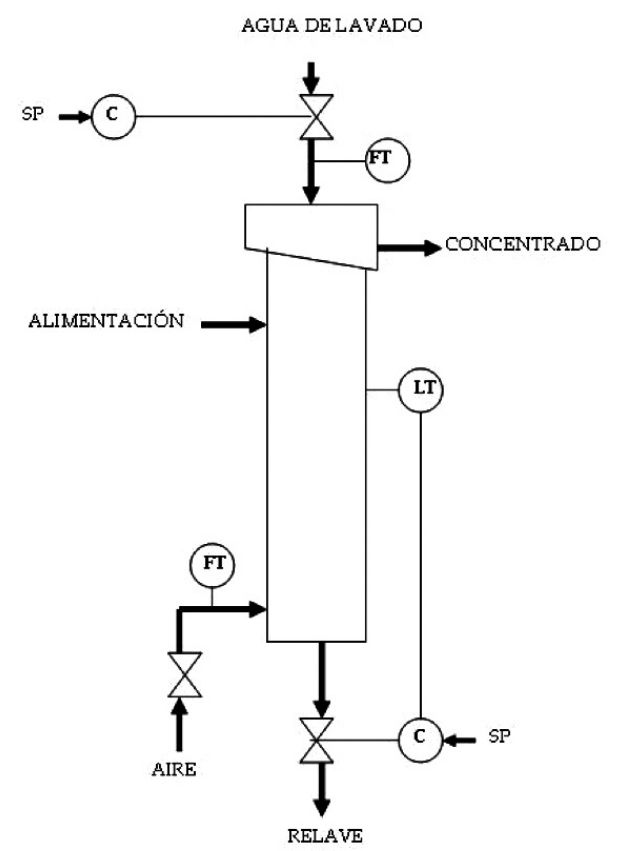

Figura 1. Esquema columna de flotación piloto con su sistema de control y la instrumentación asociada a este.

Figure 1. Scheme of the pilot flotation column system and the control instrumentation associated with this.

de la columna piloto, la frecuencia de adquisición de datos y su origen.

La velocidad superficial para un componente $i$ de flujo volumétrico $Q_{i}$ está dada por la expresión (1):

$$
J_{i}=\frac{Q_{i}}{36 \cdot A_{\text {Columna }}}
$$

Con, $i=g$ (aire), $P$ (pulpa), B (Bias), WW (agua de lavado), etc.

En una primera etapa se realiza un análisis de la data adquirida, con el objetivo de filtrar aquellos que se encuentran fuera de rango y dejar los metalúrgicamente adecuados (flujo positivo $\left(\mathrm{Q}_{\mathrm{B}}>0\right)$ y $\mathrm{L}_{\mathrm{Conc}}>$ $\left.\mathrm{L}_{\text {Alim }}\right)^{[4]}$.

Una vez que la data es filtrada, se realiza la caracterización de la operación de la columna, determinando el tiempo medio de residencia de los relaves en la zona de colección de la columna. El tiempo medio de residencia de los relaves puede ser considerado como similar al tiempo medio de residencia de la pulpa en la zona de colección de la columna. El tiempo medio de residencia $\left(T_{\text {Medio }}\right)$ fue calculado empleando la expresión (2): 
Tabla III. Listado de variables operacionales asociadas a la columna piloto

Table III. List of operational variables associated with pilot column

\begin{tabular}{|c|c|c|c|}
\hline Variable & Nombre & Significado & Frecuencia de medición \\
\hline$X_{1}$ & $L_{\text {Alim }}$ & Ley de alimentación, \% & Cada 30 min \\
\hline$x_{2}^{1}$ & $J_{\text {Allm }}$ & Velocidad superficial de agua de lavado, $\mathrm{cm} / \mathrm{s}$ & En línea, cada 1 min \\
\hline$x_{3}^{2}$ & $J^{a}$ & Velocidad superficial de aire, $\mathrm{cm} / \mathrm{s}$ & En línea, cada $1 \mathrm{~min}$ \\
\hline$X_{4}$ & $H H^{2}$ & Altura de espuma, $\mathrm{m}$ & En línea, cada 1 min \\
\hline$X_{5}^{4}$ & $C_{p}$ & Concentración de sólidos en alimentación, \% & Cada $30 \mathrm{~min}$ \\
\hline$X_{6}^{0}$ & $\mu^{p}$ & Bias, velocidad superficial de agua neto en fase espuma, $\mathrm{cm} / \mathrm{s}$ & \\
\hline & & ${ }^{*}\left(J_{\text {Aqua Relaves }}-J_{W W}\right)$ & Determinado mediante ecuación \\
\hline$x_{7}$ & $\varepsilon$ & Hold up de aire, Determinado mediante ecuación & \\
\hline$X_{8}$ & $F_{80}$ & Tamaño partícula del $80 \%$ pasante & Cada 30 min \\
\hline$X_{9}^{0}$ & $T_{\text {Medio }}$ & Tiempo medio de residencia, min & Determinado mediante ecuación \\
\hline & $X_{10}$ & $K_{F C}$ Constante de velocidad específica de flotación, $1 / \mathrm{min}$ & Determinado mediante ecuación \\
\hline
\end{tabular}

* : Velocidad superficial de agua en relaves menos velocidad superficial de agua de lavado en la alimentación.

$$
T_{\text {Medio }}=\frac{(H C-H E) \cdot(1-\varepsilon)}{J_{\text {Relaves }}}
$$

donde, HC y HE son la altura de la columna y de la espuma, respectivamente $(\mathrm{cm}), J_{\text {Relaves }}$, la velocidad superficial de relave y $\varepsilon$ es la fracción de aire (hold up de aire). Para la determinación del hold up de aire se realizó una estimación entre las principales variables que determinan este parámetro, a saber: el flujo de aire y la altura de espuma. La determinación del hold up experimental se hizo empleando el principio de desplazamiento de volumen. La figura 2 presenta la relación entre el flujo volumétrico de aire $\left(Q_{g}\right)$ y la altura de espuma (HE) de la columna de flotación.

De acuerdo a lo obtenido de la figura 2, la función que determina el hold up queda, entonces, determinada por la siguiente correlación:

$$
\varepsilon=-0,37+0,01 \cdot Q_{g}+0,05 \cdot(352-3,50 \cdot H E)(3)
$$

$$
\text { con } \quad H E=352,82-3,501 \cdot N I^{\text {Normalizada }}
$$

$$
\text { para } \quad N I^{\text {Normalizada }}=\frac{N I^{\text {Sensor }}}{\rho_{\text {Liquido }}}
$$

Para este estudio se consideró $\rho_{\text {líquido }}=1,07 \mathrm{~g} / \mathrm{cm}^{3} \mathrm{y}$ $\mathrm{NI}=107 \%$ como factores de corrección, debido al desplazamiento de las mediciones obtenidas por efecto de la densidad del medio, donde NI es el nivel

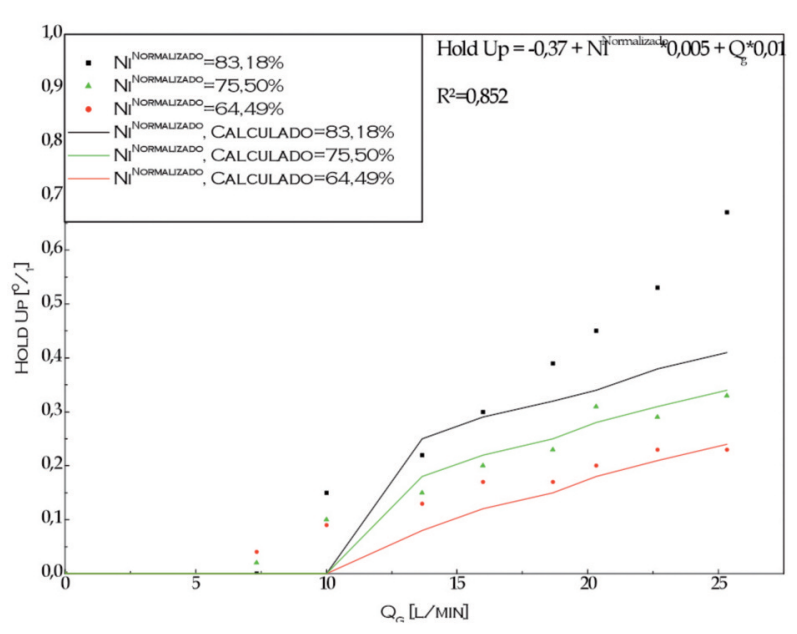

Figura 2. Relación entre Nivel de Interfaz $(N I)$, Flujo de Aire $\left(Q_{g}\right)$ en $\mathrm{m}^{3} / \mathrm{h}$ y Hold Up $(\varepsilon)$.

Figura 2. Relationship between Level Interface $(\mathrm{NI})$, Airflow $\left(Q_{g}\right)$ in $\mathrm{m}^{3} / \mathrm{h}$ and Hold Up $(\varepsilon)$.

relativo de la interfaz espuma/líquido respecto al nivel de rebose de la celda.

La data menor a $10 \mathrm{l} / \mathrm{min}$ no es considerada ya que, por condiciones operacionales, ese caudal de aire no es utilizado normalmente en este tipo de celda puesto que se debe mantener una altura adecuada de nivel de espuma en el sistema, así como una distribución de la fracción de aire (hold $u p, \varepsilon$ ) en la columna lo más eficiente posible.

Otra característica fundamental en la operación de columnas de flotación es la condición de flujo pistón, con un bajo grado de mezclamiento axial 
presente en la zona de colección. Esta condición quedará definida por el número de dispersión axial $\left(N_{D}\right)$, el cual depende de la velocidad intersticial del líquido $(\mu, \mathrm{cm} / \mathrm{s})$, ecuación (6)), y del coeficiente de dispersión axial $(D)$, ecuación (7), siendo determinado finalmente por la expresión (8):

$$
\begin{gathered}
\mu=\frac{1}{(1-\varepsilon)} \cdot\left(J_{P}+J_{B}\right) \\
D=0,063 \cdot d_{\text {columna }} \cdot\left(\frac{J_{G}}{1,6}\right)^{0,3} \\
N_{D}=\frac{D}{\mu \cdot(H C-H S-H E)}
\end{gathered}
$$

donde, $J_{P}$ y $J_{B}$ son las velocidades superficiales de pulpa de alimentación y agua de lavado neta, respectivamente (Bias), d el diámetro de la columna $(\mathrm{cm}), J_{\mathrm{G}}$ la velocidad superficial de aire y HS la altura de ubicación de los spargers (m). Como condición general, se tiene que si $D$ tiende a cero, entonces, $N_{D}$ también tiende a cero y se puede decir que la columna opera en flujo tipo pistón; en caso contrario se dice que la columna opera en flujo tipo mezcla perfecta. En este caso los datos operacionales indican una tendencia clara al flujo tipo pistón, como se muestra en la figura 3.

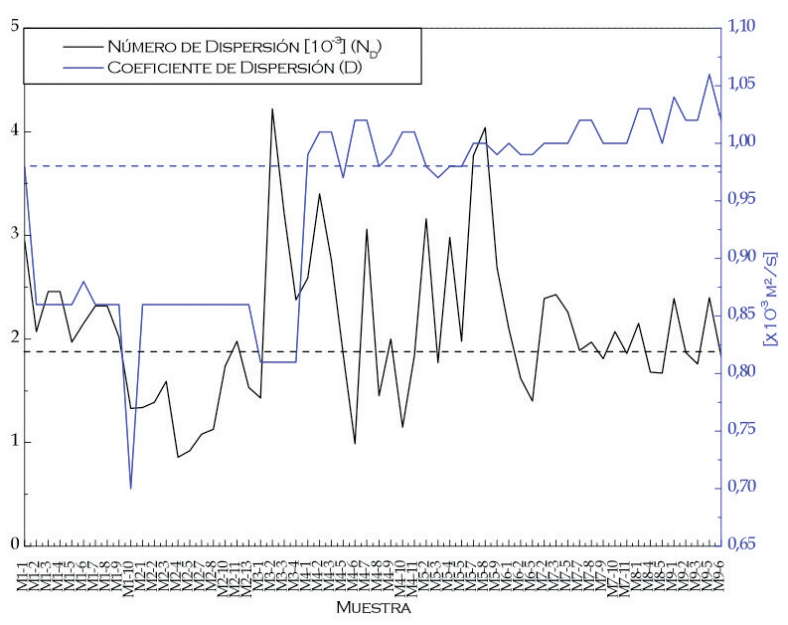

Figura 3. Coeficiente de Dispersión y Número de Dispersión característico de la columna piloto.

Figure 3. Coefficient of Dispersion and Dispersion characteristic number of the pilot column.
Teniendo esta condición determinada es posible estimar la constante de velocidad específica de un mineral sometido a flotación columnar $\left(K_{\mathrm{FC}}\right)$, a partir de la información entregada por instrumentación y la información cinética respectiva. La constante de velocidad específica de flotación queda determinada según Finch y Dobby ${ }^{[5]}$ por la relación (9):

$$
K_{F C}=\frac{1}{T_{\text {Medio }}} \cdot \operatorname{Ln}\left[\exp \left(K_{\text {Rougher }} \cdot T_{\text {Medio }}\right) \cdot R_{F}+1-R_{F}\right]
$$

donde, $K_{\text {Rougher }}$ es la constante cinética de flotación Rougher en celdas estándares y $R_{F}$ es la recuperación de la espuma en la columna. La recuperación metalúrgica $(\mathrm{Rec})$ de la especie de interés en la zona espuma varía entre 40 y $60 \%$. Una vez que se ha determinado esta constante, para cada una de las muestras es posible desarrollar modelos semi-empíricos de cada variable en relación a los parámetros, como el que se muestra en la figura 4.

En la figura 4 la relación entre $J_{g}$ y $R E$ responde a una función del tipo $Y=\left(X_{i}-X_{i}^{*}\right)^{\alpha}$, donde $X_{i}$ es la variable del parámetro $i$ y $X_{i}^{*}$ el valor máximo que se espera alcanzar de ella y $\cdot$ el grado del polinomio de esa variable, esto permite dividir el comportamiento de la curva en dos zonas, una zona ascendente (antes de $X_{i}^{*}$ ) y otra descendente (después de $X_{i}^{*}$ ). Siguiendo este procedimiento se logra desarrollar los modelos para la razón de enriquecimiento, los cuales se muestran en la tabla IV.

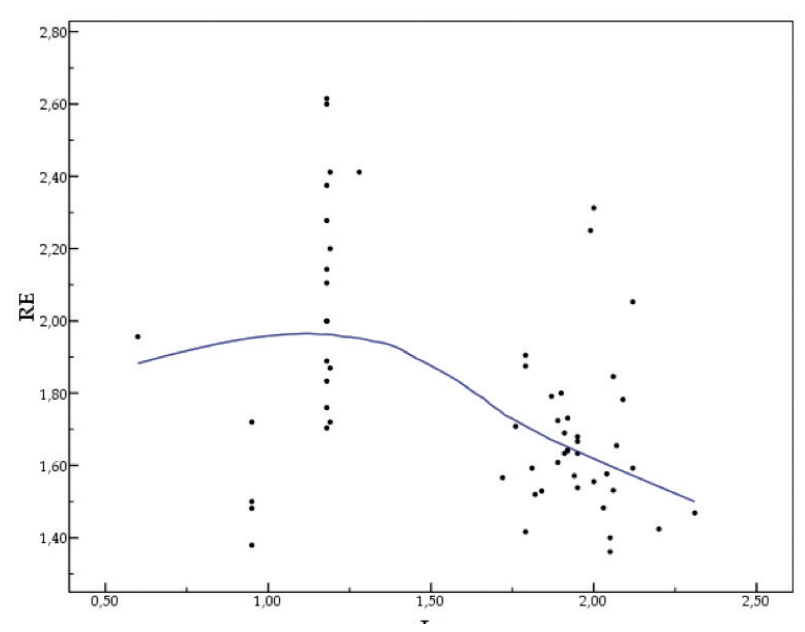

Figura 4. Relación entre Velocidad Superficial de Aire $\left(J_{g}\right)$ v/s Razón de Enriquecimiento $(R E)$.

Figura 4. Relationship between Surface Velocity Air $\left(J_{g}\right)$ versus Enrichment Ratio (RE). 
Tabla IV. Modelos matemáticos para Razón de Enriquecimiento

Table IV. Mathematical models for enrichment ratio

\begin{tabular}{cc}
\hline Para... & Se crea... \\
\hline $\mathrm{X}_{11}: H E$ & $a_{11} \cdot(H E-0,85)^{2}$ \\
$\mathrm{X}_{12}: \varepsilon$ & $\varepsilon_{12} \cdot(\varepsilon-0,25)^{2}$ \\
$\mathrm{X}_{13}: J_{G}$ & $a_{13} \cdot\left(J_{G}-1,25\right)^{2}$ \\
$\mathrm{X}_{14}: J_{B}$ & $a_{14} / J_{B}$ \\
$\mathrm{X}_{15}: F_{80}$ & $a_{15} \cdot F_{80}$ \\
$\mathrm{X}_{16}: T_{\text {Medio }}$ & $a_{16} / T_{\text {Medio }}$ \\
$\mathrm{X}_{17}: C_{p}$ & $a_{17} \cdot\left(0,24-C_{p}\right)^{2}$ \\
\hline
\end{tabular}

En la tabla V se presentan los modelos empíricos desarrollados para el modelo de $\mathrm{L}_{\text {Relaves }}$. Estos modelos empíricos complementarán a las variables operacionales presentadas en la tabla III, buscando crear, de esta forma, modelos matemáticos que se vean influenciados tanto por relaciones directas como por relaciones empíricas ${ }^{[6]}$.

El método empleado es un tipo de regresión lineal llamado Stepwise Regression ${ }^{[7]}$ que posee la particularidad de evaluar el "peso" de una variable dentro de un modelo por medio del coeficiente de regresión lineal, $\mathrm{R}^{2}$, y determinar si es válida o no la presencia de esta en la formulación del modelo.

Este método trabaja con regresiones lineales, pero se sabe que estas no responden al comportamiento de los equipos del proceso de flotación el cual es no lineal. Por lo cual, es necesario realizar un desarrollo logarítmico que si represente este proceso, la representación queda entonces de la siguiente forma:

Tabla V. Modelos matemáticos empíricos para Ley de Relaves

Table V. Mathematical models for tailings law

\begin{tabular}{cc}
\hline Para... & Se crea... \\
\hline $\mathrm{X}_{18}: H E$ & $a_{18} / H E$ \\
$\mathrm{X}_{19}: \varepsilon$ & $a_{19} \cdot(0,23-\varepsilon)^{2}$ \\
$\mathrm{X}_{20}: J_{G}$ & $a_{20} \cdot\left(1,25-J_{G}\right)^{2}$ \\
$\mathrm{X}_{21}: J_{B}$ & $a_{21} \cdot J_{B}$ \\
$\mathrm{X}_{22}: F_{80}$ & $a_{22} \cdot\left(F_{80}-44,50\right)^{2}$ \\
$\mathrm{X}_{23}: T_{\text {Medio }}$ & $a_{23} \cdot T_{\text {Medio }}$ \\
$\mathrm{X}_{24}: C_{p}$ & $a_{24} \cdot\left(C_{p}-0,22\right)^{2}$ \\
\hline
\end{tabular}

$$
Y=X_{1}^{\alpha} \cdot X_{2}^{\beta} \cdot \ldots \cdot X_{n}^{m} \Rightarrow \operatorname{Ln}(Y)=\alpha \cdot \operatorname{Ln}\left(X_{1}\right)+\beta \cdot \operatorname{Ln}\left(X_{2}\right)+\ldots m \cdot \operatorname{Ln}\left(X_{n}\right)
$$

Donde, $\alpha, \beta, \mathrm{m}$ son los coeficientes del polinomio. Empleando este método se logra crear un modelo matemático empírico que entregue resultados no lineales empleando un método de regresión lineal como el ya antes mencionado.

\section{RESULTADOS}

\subsection{Modelo empírico de RE}

Después de realizar las regresiones se llegó a determinar el modelo de RE, el cual tiene como estructura matemática:

$$
R E=\frac{H E^{0,13}}{L_{A \lim }^{0,61} \cdot T_{\text {Medio }}^{0,12} \cdot J_{B}^{0,06} \cdot F_{80}^{0,08} \cdot C_{P}^{0,09}}
$$

donde, $F_{80}$ es el tamaño de partícula pasante $80 \%$ y $\mathrm{C}_{P}$ es la concentración de sólidos de la pulpa, ambos en la alimentación. Este modelo presenta un ajuste del $90 \%$ para los datos empleados y su comportamiento se puede ver en la figura 4 .

\subsection{Modelo empírico $L_{\text {Relaves }}$}

$\mathrm{L}_{\text {Relaves }}$ queda determinada mediante el modelo:

$$
L_{\text {Relaves }}=\frac{L_{A \lim }^{1,029} \cdot H E^{0,105}}{K_{F C}^{0,098} \cdot T_{\text {Medio }}^{0,077}}
$$

El modelo posee un ajuste del $93 \%$ para los datos empleados y su comportamiento se puede ver en la figura 5.

\subsection{Modelo empírico $L_{\text {Conc }}$ \\ $L_{\text {Conc }}$ queda determinada por:

$$
L_{\text {Conc }}=R E \cdot L_{\text {Alim }}
$$

donde, $R E$ es la razón de enriquecimiento y $L_{\text {Alim }}$ la ley de alimentación. Este modelo se ajusta a un 74 \% de los datos empleados, la baja en el nivel de ajuste se 


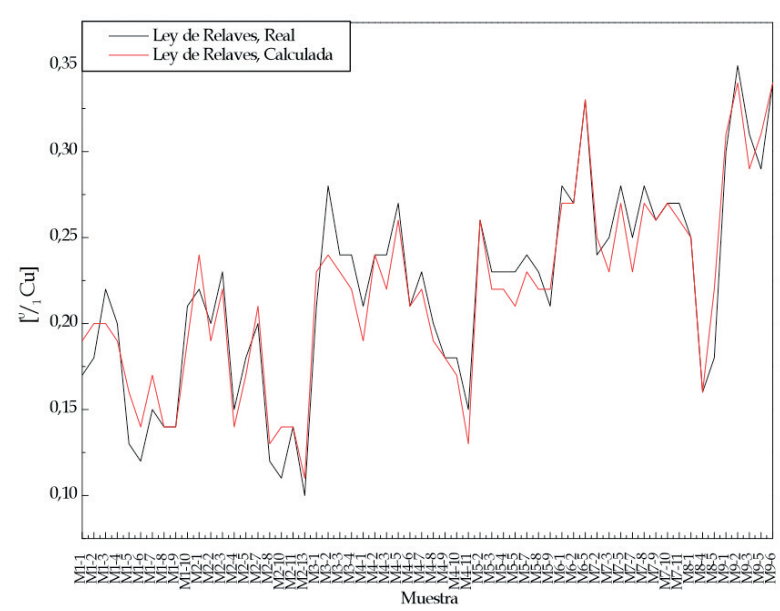

Figura 5. Comparación entre Ley de Relaves Real y el Modelado.

Figure 5. Comparison between real results of tailing law versus modeling results of them.

explicaría principalmente por posibles diferencias en la mineralogía de alimentación empleada en los ensayos y que ya se evidenciaba según lo indicado en las tablas I y II. El comportamiento de este modelo se puede ver en la figura 6 .

\subsection{Modelo empírico de Rec}

Rec queda determinada por el modelo:

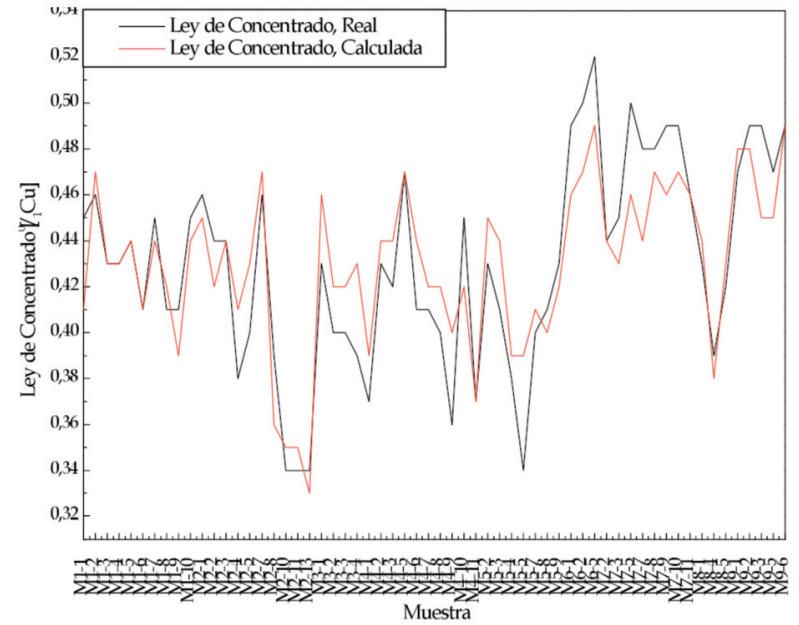

Figura 6. Comparación entre Ley de Concentrado Real y el Modelado.

Figure 6. Comparison between real results of the concentrate versus modeling results of them.

$$
\operatorname{Rec}=\frac{\left(L_{\text {Alim }}-L_{\text {Relave }}\right)^{0,83}}{L_{\text {Alim }}{ }^{0,58} \cdot\left(L_{\text {Conc }}-L_{\text {Relave }}\right)^{0,46}}
$$

Este modelo se ajusta a un $93 \%$ de los datos y su comportamiento se presenta en la figura 7.

\section{CONCLUSIONES}

El modelo empírico obtenido de RE responde de manera eficiente, siendo las principales variables que la afectan la altura de espuma y el tiempo medio de flotación.

El modelo empírico obtenido de $\mathrm{L}_{\text {Relaves }}$ responde de manera concordante con lo esperado teóricamente ${ }^{[7]}$, siendo la principal variable la altura de espuma.

Los modelos empíricos de $\mathrm{L}_{\text {Conc }}$ y Rec que se desprenden de forma directa de los modelos empíricos anteriores y que se deducen directamente de un balance de los finos de la columna, entregan información que es concordante con el comportamiento de las variables manipuladas.

El método de la regresión por pasos (Step Wise Regression $)^{[7]}$ representa una muy buena herramienta para correlacionar las variables de proceso y obtener modelos empíricos que caractericen el comportamiento de columnas de flotación.

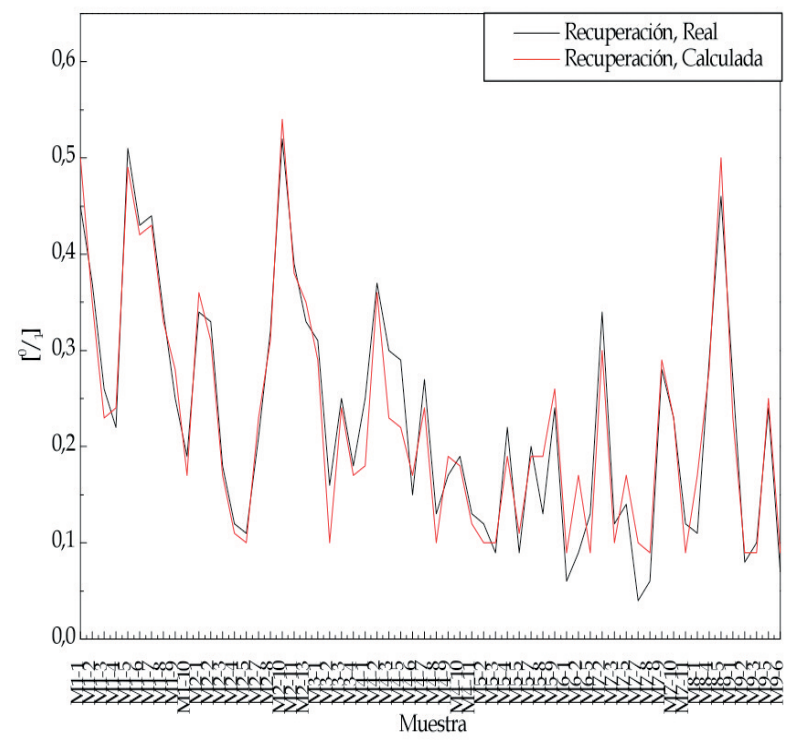

Figura 7. Comparación Entre Recuperación Metalúrgica Real y el Modelado.

Figure 7. Comparison between real results of the metallurgical recovery versus modeling results of them. 


\section{REFERENCIAS}

[1] A. Azanero, P. Nuñez, E. León y M. Morales, Rev. Inst. Investig. Fac. Minas Metal Cienc. Geogr. 11 (2003) 82-90.

[2] J. Bouchard, A. Desbiens y R. del Villar, Miner. Eng. 18 (2005) 709-720.

[3] J. Yianatos, Flotación de Minerales, Departamen to de Procesos Químicos, Biotecnológicos y Ambientales, Universidad Técnica Federico Santa María, Viña del Mar, Chile, 2005, pp. 62-87.

[4] J. Bouchard y A. Desbiens, Proc. Copper/Cobre 2003, Int. Conf. Vol 3, Santiago, Chile, 2003,
C. Gómez y C.A. Barahona (Eds.), Instituto de Ingenieros de Minas, Santiago, Chile, 2003, pp. 351-364.

[5] J.A. Finch, y G.A. Dobby, Column Flotation, Pergamon Press, Oxford, Inglaterra, 1990, pp. 180-200.

[6] O. Levenspiel, Ingeniería de las Reacciones Químicas, $2^{\underline{a}}$ Edición, Ed. Reverté, Barcelona, España, 1986, pp. 45-96.

[7] DataStep Development, SPSS Step-by-step, Tutorial for SPSS 11.5", www.datastep.com/ SPSSTutorial_1.pdf;www.datastep.com/SPSSTuto rial_2.pdf. Enero 2004. 